\title{
DEVELOPMENT OF SURFACE-WAVE DISPERSION AND ATTENUATION MAPS AND IMPROVED METHODS FOR MEASURING SURFACE WAVES
}

\author{
Jeffry L. Stevens, Jeffrey W. Given, G. Eli Baker, and Heming Xu \\ Science Applications International Corporation \\ Sponsored by Air Force Research Laboratory \\ Contract No. FA8718-05-C-0023
}

\begin{abstract}
The purpose of this new project is to optimize the procedures for measuring surface waves, particularly at regional and local distances. An important part of the project is the development of global regionalized dispersion and attenuation maps, with a particular focus on determining attenuation maps for Eurasia in the 8-15 second period band. This project follows earlier work to determine global earth models and dispersion maps using tomographic inversion of a very large data set. In this project, we are revising the procedure for performing tomographic inversion to incorporate scattering and diffraction. In addition to improving the dispersion models, this also provides a means to derive corrections for amplitude variations caused by heterogenous earth structure. We are assembling a data set of attenuation measurements through a combination of new measurements and existing measurements from other researchers, and performing tomographic inversions for $\mathrm{Q}$ structure. The inversion technique is very similar to that used for dispersion, using tomographic inversion of attenuation measurements to determine regionalized Q models which can then be used to generate attenuation maps at all frequencies (constrained by the frequency content of the data and background Q models).

We define a path corrected time-domain magnitude, which combines the time-domain narrow-band surface-wave magnitude procedure of Russell (2004) with the path corrected spectral magnitude of Stevens and McLaughlin (2001). Both the path corrected spectral magnitude and path corrected time-domain magnitude can be used reliably at any distance range, including regional and local distances. The earth structure and attenuation models described above are used to predict and correct for the amplitude variations along any source to receiver path. The procedure therefore results in an optimized surface-wave measurement procedure, which with good path corrections is valid at all distances. It is also valid at all frequencies with the caveat that frequency variations in source spectra must be taken into account if the procedure is used for Ms:mb discrimination.
\end{abstract}




\section{OBJECTIVE}

The objective of this project is to optimize the procedures for measuring surface waves, particularly at regional and local distances. An important part of the project is the development of global regionalized dispersion and attenuation maps, with a particular focus on determining attenuation maps for Eurasia in the 8-15 second period band.

\section{RESEARCH ACCOMPLISHED}

\section{Overview}

This is a new project and work to date has focused on implementing some of the procedures necessary to predict amplitude variations due to earth structure in a three-dimensional earth. An overview of the project is shown in Figure 1. In an earlier project we developed global, regionalized dispersion models that allow the phase and group velocity to be calculated between any two points on the earth. We did this by accumulating a large data set consisting of more than 1 million dispersion measurements derived by a number of researchers, and then inverting this data set to determine earth structure, which in turn is used to generate dispersion maps at all frequencies.

Figure 1. Overview of the surface wave dispersion and attenuation project.

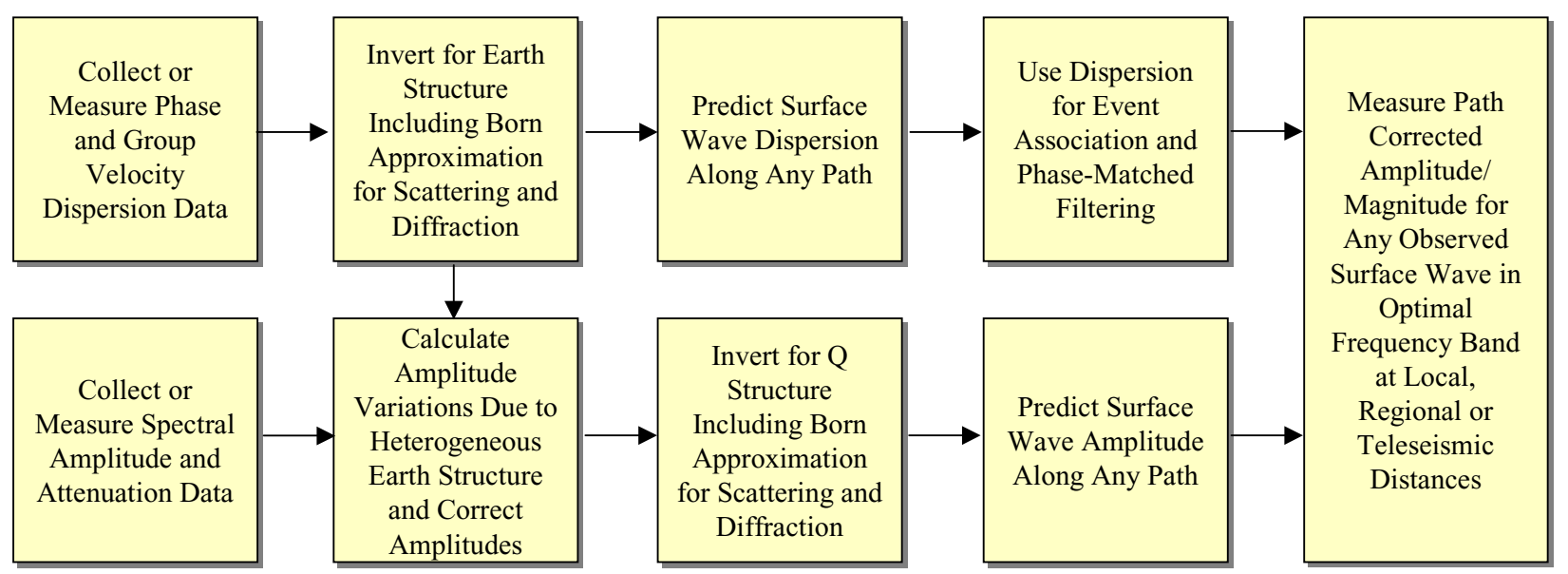

In the current project, we continue development of dispersion models, but incorporate into the modeling more complex phenomena caused by propagation in heterogeneous media including scattering and diffraction. The previous project modeled surface waves in a heterogeneous earth using the following approximations: 1) surface waves propagate along great circle paths, 2) surface-wave phase and group velocities and anelastic attenuation can be modeled using a path integral between source and receiver, and 3) energy is conserved with no mode conversion across material boundaries. This approximation is quite good for large parts of the world, but the unmodeled variations become important in regions of structural complexity.

A major emphasis of the project is the development of regionalized attenuation models. Our current model distribution includes attenuation maps as well as dispersion maps, but they are based on relatively generic models, and do not take into account attenuation differences between regions. The procedures that we developed for inversion of dispersion data for earth structure can also be applied to inversion of attenuation data for $\mathrm{Q}$ structure. The Q structures can then be used to predict attenuation between any two points on the earth.

Development of attenuation models is more difficult than development of dispersion models because it is more difficult to make attenuation measurements, and because amplitudes are more sensitive than dispersion to lateral variations in earth structure. Most of the measurements in our existing data set are group velocity measurements, which (except for very large events) can be derived accurately from single station measurements using the known origin time and location and the arrival times of different frequencies at the recording station. Phase velocities are more difficult to measure because they require either two station measurements or an assumption about the initial phase of the source. Attenuation measurements are more difficult yet, because they require either two station measurements or knowledge about the source amplitude and phase in order to determine the attenuation at the receiver point, and may also either require a correction or be unusable because of focusing/defocusing. 
In the current project, we are developing a data set of attenuation measurements. We are requesting data from other researchers performing surface-wave attenuation studies, and will make additional measurements to augment these. We supplement these measurements with Q models that have been developed by other researchers where available. As with the dispersion modeling, we invert the attenuation measurements at all frequencies, together with existing $Q$ models, for Q structure on a finite set of earth models, and then use these models to derive attenuation coefficients at all frequencies.

In addition to development of dispersion and attenuation models, we are developing improved procedures for surface wave measurement. This is particularly important at regional distances, where a traditional 20 -second $\mathrm{M}_{\mathrm{s}}$ measurement is difficult or impossible to make. There is a common misconception that surface waves cannot be measured at close distances, but in fact surface waves can be measured very close to the source and their highest signal to noise ratio is highest at the closest distances. The measurement threshold for surface waves can therefore be reduced significantly by making close in measurements; the difficulty is measuring the surface wave in a manner consistent with the measurement of more distant surface waves.

In our previous project we implemented and tested the use of path corrected spectral magnitudes. A path corrected spectral magnitude is the logarithm of the spectral amplitude of a (usually phase-matched filtered) surface wave divided by the Green's function of an explosion-generated surface wave along the same path. Doing this flattens the spectrum, and the path corrected spectral magnitude can then be determined by averaging the ratio over the optimum frequency band. The optimum frequency band depends on two things: the best signal-to-noise ratio and the best discrimination performance. At short ranges the signal-to-noise ratio is best at higher frequencies, but discrimination performance in general is better at lower frequencies. In our previous project we evaluated some of these tradeoffs and concluded that surface wave measurements for earthquake/explosion discrimination perform best at periods greater than 10 seconds.

In the current project we will be improving the path corrected spectral magnitude using the attenuation maps and amplitude corrections developed in this project. At very close distances ( $<5$ degrees) surface wave attenuation is generally small and amplitude variations may result more from heterogeneous earth structure than from attenuation. We can test this by assessing whether the amplitude corrections developed from the earth models lead to more consistent measurements for these very short paths.

We are also developing an improved path corrected time-domain magnitude, which provides a way to measure a regionalized time-domain $\mathrm{M}_{\mathrm{s}}$ that is consistent in value with a traditional time-domain $\mathrm{M}_{\mathrm{s}}$ even at very close distances. This is accomplished by combining the path corrected spectral magnitude described above with a time-domain $\mathrm{M}_{\mathrm{s}}$ procedure developed by Russell (2004). The Russell procedure is a path corrected time-domain magnitude for an earth average path. We can improve this by correcting for regional variations in earth structure and attenuation. 


\section{The inversion procedure for the 3D earth model}

In our previous projects, we inverted a large volume of dispersion data for global earth structure. Global earth structure refers to a set of vertically layered earth models defined for each cell of a one-degree by one-degree grid of the earth. This procedure is summarized here, and in the following sections we show how it can be modified to include scattering and diffraction and modified to invert attenuation data for global Q structure. The relationship between dispersion and the shear wave velocities of the layers in the earth model is non-linear, so the shear velocities are estimated by an iterative least squares inversion procedure. At each step a system of equations is formed, augmented by additional equations of constraint, and then solved by the LSQR algorithm. The equations solved are

$$
\left(\begin{array}{c}
A \\
s H \\
\lambda I
\end{array}\right) \overrightarrow{\Delta x}=\left(\begin{array}{c}
\overrightarrow{\Delta d} \\
-s H \vec{x} \\
\lambda\left(\vec{x}_{C}-\vec{x}\right)
\end{array}\right)+\vec{\varepsilon}
$$

where $\Delta \mathbf{x}$ is the vector of adjustments to the shear wave slownesses of layers in each of the 577 model types. $\Delta \mathbf{d}$ is the vector of slowness differences between predicted and observed dispersion measurements. $\varepsilon$ is the vector of residuals that remain after inversion (the inversion minimizes $|\varepsilon|)$. $\mathbf{x}$ is the vector of slownesses estimated in the previous iteration. The elements of the matrix A consist of partial derivatives of dispersion predictions with respect to shear wave slownesses in each layer. $\mathbf{H}$ is a difference operator that applies to vertically neighboring layers and has the effect of constraining the vertical smoothness of velocity profile. $\mathbf{H}$ applies to layers in the crust and upper mantle, but has explicit discontinuities at the crust/mantle boundary and at the base of surface sediments. The weighting of the smoothness constraint is $\mathbf{s}$ and can be a diagonal matrix (for variably weighted smoothing) or a scalar (constant smoothing). $\mathbf{I}$ is the identity matrix and $\lambda$ weights the damping which constrains the norm of the difference between final slownesses and constraining model slownesses $\mathbf{x}_{\mathbf{c}}$ (in this case a variant of the Crust 2.0 values). $\lambda$ can be a scalar for constant damping, or a diagonal matrix for variable damping.

\section{Correction for scattering and diffraction due to a realistic heterogeneous earth model}

In our previous projects, we based the methodology for dispersion analysis and amplitude prediction on an approximation originally due to McGarr (1969) that uses propagation of surface waves along great circle paths with conservation of energy across material interfaces and no mode conversion. With these approximations, surface-wave propagation in a heterogeneous, anelastic structure takes the following form, separating source, path and receiver (notation follows Harkrider et al., 1994):

$u_{z}(\omega, r, \varphi)=\frac{1}{\sqrt{a_{e} \sin \left(r / a_{e}\right)}} \sqrt{\frac{2 A_{R_{1}}}{\pi \omega c_{1}{ }^{2}}} \sqrt{c_{2} A_{R_{2}}} \exp \left[i\left(\pi / 4-\omega r / c_{p}-\gamma_{p} r\right)\right] F_{s}(\omega, \varphi, h)$

where $\omega$ is angular frequency, $r$ is source to receiver distance, $h$ is source depth, $a_{e}$ is the radius if the earth, $\varphi$ is azimuth, $A_{R}$ is the Rayleigh wave amplitude function, $c$ is phase velocity, $\gamma$ is the attenuation coefficient, and the subscripts 1,2 , and $p$ refer to parameters derived from the source region structure, parameters derived from the receiver region structure, and parameters which are defined by path averages, respectively. All source properties are contained in the function $F_{s}$. For an isotropic explosion source, the Rayleigh wave spectrum can be written

$u_{z}\left(\omega, h_{x}, r\right)=M_{0}^{\prime} \frac{S_{1}^{x}\left(\omega, h_{x}\right) S_{2}(\omega) \exp \left[-\gamma_{p}(\omega) r+i\left(\varphi_{0}-\omega r / c_{p}(\omega)\right)\right]}{\sqrt{a_{e} \sin \left(r / a_{e}\right)}}$

where $\varphi_{0}$ is the initial phase equal to $-3 \pi / 4, S_{1}^{x}$ depends on the source region elastic structure and the explosion source depth, $S_{2}$ depends on the receiver region elastic structure. $M_{0}^{\prime}=\frac{3 \beta^{2}}{\alpha^{2}} M_{0}$ where $M_{0}$ is the explosion isotropic moment. $M_{0}^{\prime}$ is defined this way so that the function $S_{1}^{x}$ does not depend explicitly on the material properties at the source depth. (More details are given in Stevens and McLaughlin (2001) and Stevens and Murphy (2001)). 
In our experience, this approximation works very well for modeling surface wave dispersion and amplitude over most of the world. In the current project, however, we are performing a more complete analysis including effects of scattering and diffraction. This is important for two reasons:

1) Some of the remaining residual in the global dispersion models is due to scattering and diffraction, and incorporation of these effects into our analysis will allow us to correct for them; and

2) To perform inversion of attenuation data for $Q$ structure as described in the following section, we need to correct the amplitude for the effects of heterogeneous structure. The effect of heterogeneous structure on amplitude is stronger than on dispersion.

Modeling of scattering and diffraction is an active area of current research. Most of the research relevant to this project use variants of the single-scattering Born approximation to model the scattered wave field (Snieder, 1986). Zhou et al. (2004) summarize this work and derive sensitivity kernels for phase, amplitude, and arrival angle. The Born approximation models the observed surface wave at a receiver as a sum of a direct wave plus waves scattered from material inhomogeneities throughout the region. The sensitivity kernels show that the scattering and diffraction are largely confined to scatterers within the first Fresnel zone, which is defined by $k\left(\Delta^{\prime}+\Delta^{\prime \prime}-\Delta\right)<3 \pi / 4$ where $k$ is the wavenumber and $\Delta, \Delta^{\prime}, \Delta^{\prime \prime}$ are the source to receiver, source to scatterer, and scatterer to receiver distances, respectively.

Ritzwoller et al. (2002) used a simplified version of the Born approximation to include diffraction in surface-wave tomography. They modeled the sensitivity kernel with a boxcar function the width of the Fresnel zone normal to the source to receiver path, then used an area integral over this region in place of the ray theory path integral for performing tomographic inversion. Yoshizawa and Kennett (2004) and Kennett and Yoshizawa (2002) use a similar technique with a narrower kernel that they believe to be more representative of realistic surface waves. Other recent papers on scattering and implications for surface-wave tomography include Spetzler et al. (2001, 2002).

Although the methods described above differ in detail, they are similar in practice and give similar results. That is, all the methods described predict the surface wave phase velocity by integrating slowness over an area approximately equal to the Fresnel zone with similar, although not identical, kernels. These techniques provide a straightforward way to incorporate scattering and diffraction into the inversion procedure described in the previous section. The matrix $\mathbf{A}$ in equation 1 is currently calculated using a path integral to calculate the phase velocity, with each element of the matrix corresponding to a piece of the path weighted according to the fraction of the path crossing a grid block and the sensitivity of the observable to the model velocity. This can be replaced by integration over the Fresnel zone area, which changes the weighting of each element and increases the number of elements corresponding to each ray. The matrix requires more time to calculate, but the inversion procedure is the same as in the ray-based tomographic inversion.

\section{Inversion of attenuation data for $Q$ structure}

Inversion of attenuation data for $\mathrm{Q}$ structure can be accomplished using equation 4 , which has the same form as equation 1 above:

$$
\left(\begin{array}{c}
A \\
s H \\
\lambda I
\end{array}\right) \overrightarrow{\Delta x}=\left(\begin{array}{c}
\overrightarrow{\Delta d} \\
-s H \vec{x} \\
\lambda\left(\vec{x}_{C}-\vec{x}\right)
\end{array}\right)+\vec{\varepsilon}
$$

with the following changes:

1. The data are attenuation residuals instead of dispersion residuals. Attenuation estimates are derived from an existing Q model, and the differences between those and the observations are the data used in the inversion. Amplitude measurements are corrected for the effects of heterogeneous structure.

2. The matrix $\mathrm{A}$ is derived from derivatives of the attenuation coefficients with respect to model $\mathrm{Q}$ in each layer for the path-averaged inversion, and includes Born scattering sensitivity functions for the area integrals.

3. The constraining model is based on the best available Q models. 
Note that equations 2 and 3 give the equation for the distance dependence of surface waves. In equation 5 , we have rewritten this slightly to show how it is used in the inversion for attenuation. We have included the dependence on $r$ in $S_{2}$ to show that it corresponds to the earth structure at the receiver location $r$ (this provides a station dependent amplitude correction for local earth structure).

$$
U(\omega, r) \sim \frac{\left|S_{2}(\omega, r)\right| \exp \left[-\gamma_{p}(\omega) r\right]}{\sqrt{a_{e} \sin \left(r / a_{e}\right)}}
$$

The data used in equation 4, if derived, for example, from two stations along the same ray path, is calculated by taking the ratio of equation 5 evaluated at the two locations, and solving for $\gamma_{p}$. The amplitude measurements at both stations are corrected for heterogeneous structure effects prior to solving for $\gamma_{p}$.

Q models are much less well defined than the earth models used for the dispersion inversion. That is, for the dispersion inversion, we have a good background model developed from sediment maps, ocean depths, Crust 2.0, and the AK135 mantle model, but Q models are not known at the same level of detail. With the formulation of equation 4, however, we can include a generic background Q model augmented with better Q models for specific areas developed in other research projects. The generic model gives relatively distance-independent measurements at periods near and below 20 seconds. However, we expect to see much larger variations at the higher frequencies that are the primary focus of this study. 


\section{Optimization of surface wave measurement}

Surface-wave measurements traditionally have been made by measuring a time-domain amplitude at a period near 20 seconds and then calculating a surface-wave magnitude $M_{\mathrm{s}}$. This procedure is problematic at regional distances because the surface wave is not well dispersed and a distinct 20 -second arrival may not be present. It is possible to measure time-domain amplitudes at higher frequencies with corrections (e.g., Marshall and Basham, 1972), however measurements may be inaccurate due to differences in dispersion caused by differences in earth structure. Stevens and McLaughlin (2001) suggested as an alternative replacing time-domain measurements with a path corrected spectral magnitude.

\section{Path corrected spectral magnitudes}

The path corrected spectral magnitude, $\log M_{0}$, is calculated by dividing the observed surface wave spectrum by the Green's function for an explosion of unit moment (see Equation 3) and taking the logarithm of this ratio, averaged over any desired frequency band. The path corrected spectral magnitude is defined as the logarithm of:

$$
M_{0}^{\prime}=\left|U(\omega, r, \theta) /\left(\frac{S_{1}^{x}\left(\omega, h_{x}\right) S_{2}(\omega) \exp \left[-\gamma_{p}(\omega) r\right]}{\sqrt{a_{e} \sin \left(r / a_{e}\right)}}\right)\right|
$$

where $U$ is the observed surface-wave spectrum, and as above $S_{1}^{X}$ depends on the source region elastic structure and the explosion source depth, $S_{2}$ depends on the receiver region elastic structure, and $\gamma_{p}$ is the attenuation coefficient that depends on the attenuation integrated over the path between the source and receiver. All of the functions in Equation 6 are easily derived from plane-layered earth models, and allow the measurement to be regionalized to account for differences in earth structure at the source and receiver, and due to attenuation along the path.

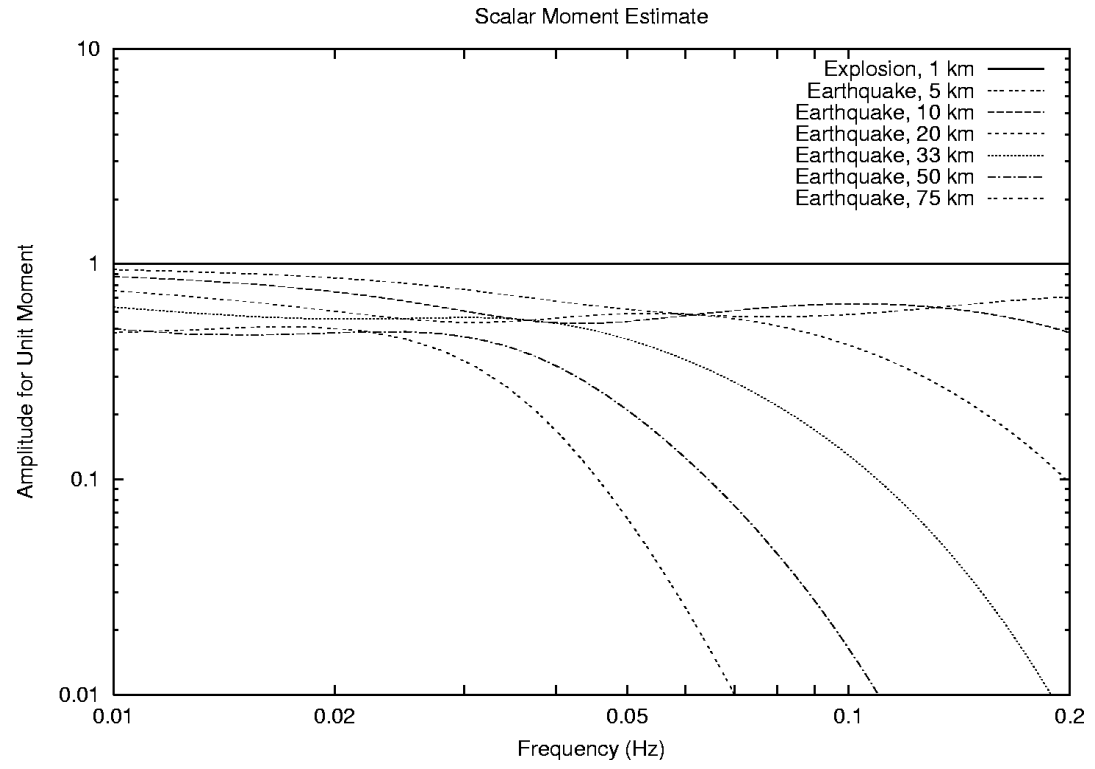

Figure 2. Path corrected spectra for an explosion and for earthquakes calculated for several depths. The path corrected explosion spectrum is flat over the entire frequency band (for perfect data and path correction), while the path corrected earthquake spectrum is flattened, but has some variation due to source mechanism and source depth.

The advantages of using $\log M_{0}$ instead of the traditional surface-wave magnitude $M_{\mathrm{s}}$ are that $\log M_{0}$ is insensitive to dispersion, independent of distance, works well at regional distances, and can be regionalized. Regionalized path corrected spectral magnitudes incorporate geographic variations in source excitation and attenuation. Furthermore, as discussed below, it can in principle be measured over different frequency bands to optimize the signal-to-noise ratio. $M_{\mathrm{s}}$ and $\log M_{0}$ share some limitations: spectra from earthquakes vary due to source mechanism and depth, and errors can occur if the measurement is made in a spectral dip or at high frequencies for a deep event (Figure 2). 
Azimuthal variations in amplitude caused by focal mechanism also affect the amplitudes of both $\log M_{0}$ and $M_{\mathrm{s}}$. $\log M_{0}$ can also be corrected for structural heterogeneity using the amplitude corrections described earlier.

\section{Path corrected time-domain magnitudes}

Russell (2004) proposed a new type of surface-wave magnitude $\mathrm{M}_{\mathrm{s}(\mathrm{b})}$ which differs from a traditional 20 second magnitude in that it uses a Butterworth filter to measure a time-domain amplitude in a narrow band around any desired frequency, and then applies a correction for the source function similar to the explosion source function used in the path corrected spectral magnitude described above. The main purpose of $\mathrm{M}_{\mathrm{s}(\mathrm{b})}$ is to allow surface waves to be measured at regional distances at higher frequencies. Bonner et al. (2004) showed that it gave consistent results in a test study. The magnitude is defined by

$$
M_{s(b)}=\log \left(A_{b}\right)+\frac{1}{2} \log (\sin \Delta)+0.0031\left(\frac{20}{T}\right)^{2.3} \Delta-0.66 \log \left(\frac{20}{T}\right)-\log \left(f_{c}\right)-0.43
$$

where $A_{b}$ is the filtered amplitude, $T$ is the measured period, and $f_{c}$ is the Butterworth filter width. It is instructive to compare the terms in the Russell magnitude with the Rezapour and Pearce $\mathrm{M}_{\mathrm{s}}$ and the path corrected spectral magnitude $\log M_{0}$ described above. This is shown in Table 1.

Table 1. Comparison of time-domain and spectral magnitude measurement and correction terms

\begin{tabular}{|c|c|c|c|c|c|c|c|c|}
$\begin{array}{c}\text { Magnitude } \\
\text { Type }\end{array}$ & $\begin{array}{c}\text { Amplitude } \\
\text { Measure }\end{array}$ & Source & Receiver & $\begin{array}{c}\text { Geometric } \\
\text { Spreading }\end{array}$ & Attenuation & Dispersion & Filter & Norm \\
\hline $\mathrm{M}_{\mathrm{s}}$ & $\log (\mathrm{A} / \mathrm{T})$ & & & $\frac{1}{2} \log (\sin \Delta)$ & $0.0046 \Delta$ & $\frac{1}{3} \log \Delta$ & & 2.37 \\
\hline $\mathrm{M}_{\mathrm{s}(\mathrm{b})}$ & $\log \left(\mathrm{A}_{\mathrm{b}}\right)$ & $-0.66 \log \left(\frac{20}{T}\right)$ & & $\frac{1}{2} \log (\sin \Delta)$ & $.0031\left(\frac{20}{T}\right)^{2.3} \Delta$ & & $-\log \left(f_{c}\right)$ & -0.43 \\
\hline $\log \mathrm{M}_{0}$ & $\log \left(\mathrm{A}_{\mathrm{s}}\right)$ & $-\log \left(\mathrm{S}_{1}\right)$ & $-\log \left(\mathrm{S}_{2}\right)$ & $\frac{1}{2} \log (\sin \Delta)$ & $\gamma_{p} \Delta$ & & &
\end{tabular}

Note that each magnitude makes a slightly different set of corrections. $\log \mathrm{M}_{0}$ corrects for both source and receiver structure based on earth models at those locations and an explosion Green's function at the source. Similarly, $\mathrm{M}_{\mathrm{s}(\mathrm{b})}$ applies a source correction based on typical explosion source excitation. The Airy phase dispersion correction accounts for superposition of waves with similar group velocities, and is needed only in the time domain. $\mathrm{M}_{\mathrm{s}(\mathrm{b})}$ uses a Butterworth filter that is sufficiently narrow to avoid this problem. The filter correction corrects for the width of the Butterworth filter. The normalization for the two $\mathrm{M}_{\mathrm{s}}$ measurements is chosen to make it consistent with historical $\mathrm{M}_{\mathrm{s}}$ magnitudes at a chosen distance range. $\log \mathrm{M}_{0}$ has natural units of $\log$ moment and is not otherwise normalized; however, Stevens and McLaughlin (2001) showed that subtracting 11.75 makes $\log \mathrm{M}_{0}$ consistent with the Rezapour and Pearce $\mathrm{M}_{\mathrm{s}}$. Attenuation for $\mathrm{M}_{\mathrm{s}}$ is an empirical correction based on a very large number of 20 second measurements. $\mathrm{M}_{\mathrm{s}(\mathrm{b})}$ similarly uses an empirical attenuation correction, but also includes an empirical correction for the change in attenuation with frequency. $\log \mathrm{M}_{0}$ uses attenuation calculated from earth (velocity, density, and Q) models along a source to receiver path. None of the magnitude measurements include any correction for scattering and diffraction.

A path corrected time-domain magnitude can be derived by combining the path corrected spectral magnitude with

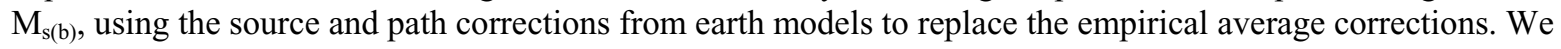
define the path corrected time-domain magnitude $\mathrm{M}_{\mathrm{s}(\mathrm{bp})}$ as:

$$
M_{s(b p)}=\log \left(A_{b}\right)+\frac{1}{2} \log (\sin \Delta)+\gamma_{p} \Delta-\log \left(S_{1}\right)-\log \left(S_{2}\right)-\log \left(f_{c}\right)+C_{b p}
$$

where $\mathrm{C}_{\mathrm{bp}}$ is a constant chosen to make $\mathrm{M}_{\mathrm{s}(\mathrm{bp})}$ consistent with historical magnitudes. Although equation 8 may appear more complicated than equation 7 , the functions $S_{1}, S_{2}$, and $\gamma_{p}$ are easily tabulated and stored in files, and a computer can quickly calculate them for any path based on a simple lookup table. There is substantial regional variation in these quantities that should be removed to ensure consistent measurements (examples of $S_{1}, S_{2}$, and $\gamma$ for 
continental and oceanic structures are shown in Stevens and McLaughlin, 1996). Another advantage of this approach is that it can ensure that $f_{c}$, which must be less than a minimum value calculated from the group velocity, is always set appropriately.

\section{CONCLUSIONS AND RECOMMENDATIONS}

We are implementing a procedure for optimizing the measurement of surface waves in the time and frequency domains using methods that model amplitude variations due to scattering and diffraction in addition to anelastic attenuation. Since the project started only recently it is premature to make specific conclusions or recommendations.

\section{REFERENCES}

Bonner, J. L., D. T. Reiter, D. G. Harkrider, and S. Russell (2004), Development of a time-domain, variable-period surface wave magnitude measurement procedure for application at regional distances, in Proceedings of the $26^{\text {th }}$ Annual Seismic Research Review, Trends in Nuclear Explosion Monitoring, Los Alamos National Laboratory document LA-UR-04-5801, Orlando, FL, September 21-24, 2004.

Harkrider, D. G., J. L. Stevens, and C. B. Archambeau (1994), Theoretical Rayleigh and Love waves from an Explosion in Prestressed Source Regions, Bull. Seism. Soc. Am. 84: 1410-1442.

Kennett, B. L. N., and K. Yoshizawa (2002), A reappraisal of regional surface wave tomography, Geophys. J. Int. 150: $37-44$.

Marshall, P. D. and P. W. Basham (1972), Discrimination between earthquakes and Underground Nuclear Explosions Employing an Improved $\mathrm{M}_{\mathrm{s}}$ Scale, Geophys. J. R. astr. Soc. 28: 431-458.

McGarr, A. (1969), Amplitude Variations of Rayleigh Waves - Propagation Across A Continental Margin, Bull. Seism. Soc. Am. 59: 1281-1305.

Ritzwoller, M. H., N. M. Shapiro, M. P. Barmin, and A. L. Levshin (2002), Global surface wave diffraction tomography, J. Geophys. Res. 107: 2335, doi:10.1029/2002JB001777.

Russell, D. R. (2004), Theoretical analysis of narrow-band surface wave magnitudes, AFTAC technical report AFTAC-TR-04-004.

Snieder, R. (1986), 3-D linearized scattering of surface waves and a formalism for surface wave holography, Geophys. J. R. astr. Soc. 84: 581-605.

Spetzler, J., J. Trampert and R. Snieder (2001), The effect of small-scale heterogeneity on the arrival time of waves, Geophys. J. Int., 145, 786-796.

Spetzler, J., J. Trampert and R. Snieder (2002), The effect of scattering in surface wave tomography, Geophys. J. Int. 149, 755-767.

Stevens, J. L. and K. L. McLaughlin (1996), Regionalized Maximum Likelihood Surface Wave Analysis, Maxwell Technologies Technical Report submitted to Phillips Laboratory, PL-TR-96-2273, SSS-DTR-96-15562, September.

Stevens, J. L. and K. L. McLaughlin (2001), Optimization of surface wave Identification and measurement, Pure and Applied Geophysics 158: 1547-1582.

Stevens, J. L. and J. R. Murphy (2001), Yield Estimation from surface wave amplitudes, Pure and Applied Geophysics 158: 2227-2251.

Yoshizawa, K., and B. L. N. Kennett (2004), Multimode surface wave tomography for the Australian region using a threestage approach incorporating finite frequency effects, J. Geophys. Res. 109: B02310, doi:10.1029/2002JB002254.

Zhou, Y., F. A. Dahlen and G. Nolet (2004), Three-dimensional sensitivity kernels for surface wave observables, Geophys. J. Int. 158: 142-168. 